
\title{
25 Research Soure \\ The Valuable Role of Armc1 in Invasive Breast Cancer as a Novel Biomarker
}

\section{Yunhao Gan}

Chongqing Medical University

Fuxin Zhong

Chongqing Medical University

Lingyu Li ( $\nabla 102815 @ c q m u . e d u . c n)$

Chongqing Medical University

Hao Wang

People's Hospital of Yubei District of Chongqing

\section{Research Article}

Keywords: Invasive breast carcinoma, Armadillo repeat-containing 1, Biomarker

Posted Date: January 5th, 2022

DOI: https://doi.org/10.21203/rs.3.rs-1185959/v1

License: (c) (1) This work is licensed under a Creative Commons Attribution 4.0 International License. Read Full License

Version of Record: A version of this preprint was published at BioMed Research International on March 26th, 2022. See the published version at https://doi.org/10.1155/2022/1740295. 


\section{Abstract}

Background: Invasive breast carcinoma (BRCA) is a common type of breast cancer with high incidence in clinics, so it is significant to find an effective biomarker for BRCA diagnosis and treatment. Although some Armadillo (Arm)-repeat proteins families are confirmed to be biomarkers in cancers, the role of Armadillo repeat-containing 1 (ARMC1) in BRCA remains unknown.

Methods: We analyzed the ARMC1 expression in normal breast tissues and BRCA samples, and its association with overall survival by the public database. $\chi^{2}$ test evaluated the risks associated with ARMC1 expression in TCGA-BRCA patient samples. The ARMC1 mutations in BRCA were explored in the cBioportal database. Besides, the GO and KEGG analysis was used to explore the potential signaling pathways of ARMC1 in BRCA. Lastly, Immunohistochemistry and immunohistochemistry were performed to validate the ARMC1 expression in BRCA.

Results: ARMC1 level in tumor sample was significantly higher than that in normal tissue, and it was also related to lower survival. The factors in clinical patients such as tumor stage and grade and histology were associated with ARMC1 expression. There were $32 \%$ of ARMC1 genetic mutations in BRCA, and the amplification and high expression made up the majority of them. Also, ARMC1 might regulate BRCA by involving in the cell cycle. Increased ARMC1 expression was found in clinical breast carcinoma tissues by our confirmatory experiments.

Conclusions: All the results revealed that ARMC1 may play a significant role in BRCA as a biomarker, it provides valuable clues for the treatment and diagnosis of invasive breast cancer.

\section{Background:}

Breast cancer is one of the most prevalent cancers among female patients, and it is also the main cause of cancer death in women [1, 2]. Diagnostic techniques including mammography and magnetic resonance imaging are the primary approaches for diagnosing breast cancer in clinical practice [3]. In terms of treatment, radical mastectomy, radiotherapy and chemotherapy were the preferred treatments for breast carcinoma [4]. With the improvement of screening ability and detection technology, the diagnosis and therapy for BRCA have made ideal progress in recent years [5]. However, a large number of patients are still diagnosed with the BRCA each year, it is also the second common cause of cancer death in women [6]. Therefore, exploring a novel biomarker remains to be crucial for BRCA research.

In search of potential biomarkers for BRCA, we discovered the Armadillo (Arm)-repeat proteins family widely involved in cancer progression. According to the Arm family studies, they have diverse works in many eukaryotes through their Arm-repeats structure [7]. Several Arm members have been demonstrated to be involved in tumor development and invasiveness. As a famous member of the Arm family, increased $\beta$-catenin expression in the cytoplasm and nuclear transfer can promote pro-oncogenes transcription such as c-Myc and CyclinD- 1 by the Wnt/ $\beta$-catenin signaling pathway $[8,9]$. In addition, the interaction between ARMC12 and retinoblastoma binding protein 4 can promote neuroblastoma 
progression by inhibiting the transcription of tumor suppressor genes [10]. However, Some Arm family members have a role in inhibiting cancer. Adenoma polyposis coli (APC) can degrade the $\beta$-catenin through glycogen synthase kinase $3 \beta$ (GSK3 $\beta$ ), thereby blocking Wnt/ $\beta$-catenin cancer signaling in colorectal cancer [11].

Armadillo repeat-containing 1 (ARMC1) was originally found in mitochondrion as a structural subunit [12]. Its gene locates at position 8q13.1 of the human chromosome. The similar repeating 42 amino acid motif is the characteristic of it as an Arm family member. However, the role of ARMC1 in cancers remains unclear, so it is of great significance to study the role of ARMC1 in breast cancer.

This study was to explore whether ARMC1 acts as a valuable biomarker for BRCA. In our study, we identify ARMC1 can lead to a poor prognosis for BRCA patients by exploring ARMC1 expression in BRCA samples and evaluating the risks in TCGA-BRCA patients. We also found ARMC1 genetic mutations in BRCA and the role of ARMC1 co-expressed genes in cell cycle regulation. At last, we identified the higher ARMC1 level in clinical breast tissues. This study was to explore whether ARMC1 acts as a valuable biomarker for BRCA.

\section{Methods:}

\section{Gene Expression Profiling Interactive Analysis}

The Gene Expression Profiling Interactive Analysis (GEPIA, http://gepia.cancer-pku.cn/) is a web-analysis tool based on TCGA and GTEx data, which provide interactive and customizable functions including differential expression analysis, profiling plotting, correlation analysis, and patient survival analysis [13]. GEPIA database was used to explore ARMC1 expression and its overall survival in BRCA samples.

\section{Kaplan-Meier plotter}

Kaplan-Meier plotter (http://kmplot.com/analysis/) is a widely used online-analysis tool for prognostic value with plentiful gene expression data and relevant clinical data [14]. Kaplan-Meier plotter was performed to analyze the prognostic value of ARMC1 in BRCA.

\section{Human Protein Atlas}

Human Protein Atlas (HPA) database (https://www.proteinatlas.org) is a tool for validating immunohistochemistry-based protein expression patterns and extending the map of protein expression patterns in cancer research projects [15]. HPA was used to search the protein and mRNA expression levels of ARMC1, and analyze the prognostic evaluation of ARMC1 in BRCA.

\section{The Cancer Genome Atlas}


The Cancer Genome Atlas (TCGA, https://portal.gdc.cancer.gov/) is a public database that provides large cancer genomic profiles of over 30 human tumors, its primary purpose is to research individual cancer types through large-scale genome sequencing and integrated multi-dimensional analyses[16]. TCGA database was used to collect the BRCA cases with ARMC1 differentially expression for prognostic evaluation and risk factor analysis. a total of 869 BRCA patients were available in TCGA, and the full clinical dataset also was downloaded.

\section{cBio Cancer Genomics Portal}

The cBio Cancer Genomics Portal (http://cbioportal.org) is an open-access database, which provides more than 5,000 tumor samples from 20 cancer studies to explore multidimensional cancer genomics data sets [17]. The cBio Cancer Genomics Portal database was used to find the genetic mutations of ARMC1 in BRCA and co-expressed genes associated with ARMC1.

\section{Metascape}

Metascape (metascape.org/gp/) is a web-based portal that combines works including functional enrichment, interactome analysis, gene annotation, and membership search [18]. Metascape was used to analyze the signaling pathways based on the co-expressed gene data related to ARMC1 expression.

\section{DAVID Functional Annotation Tool}

DAVID Bioinformatics Resources (DAVID/https://david.ncifcrf.gov/) is a functional annotation tool with public bioinformatics resources. It Provides over 40 available annotation categories at the gene and protein level [19]. DAVID was used to performing the GO term and KEGG analysis by the co-expressed genes associate with ARMC1.

\section{Patient selection}

According to the situation that ARMC1 is mainly mutated in invasive ductal carcinoma and invasive lobular carcinoma, corresponding BRCA cases (10 of invasive ductal carcinoma, 5 of invasive lobular carcinoma) that recently appeared in clinical practice were randomly selected. Fifteen pairs of paraffin sections including BRCA tissues and corresponding para-carcinoma tissues were obtained from the Pathological Diagnosis Center of Chongqing Medical University (Chongqing, China). All samples for the confirmatory experiment were selected female patients, and their ages range from 33 to 66 years old. Pathological diagnosis of breast cancer types included invasive ductal carcinoma cases and invasive lobular carcinoma. More characteristics about participants as shown in Supplement Table 1. 


\section{Cell line and their extraction}

Human breast carcinoma cell lines (MCF-7) and Human normal breast cells (MCF-10A) were used for in vitro experiments, which were purchased from Procell Life Science\&Technology (Wuhan, China). MCF-7 cells were maintained in Dulbecco's Modified Eagle Medium (DMEM; Gibco, Carlsbad, USA) supplemented with $10 \%$ fetal bovine serum (FBS) and $10 \mu / \mathrm{ml}$ penicillin G/streptomycin, and MCF-10A were maintained in 10A cell-specific culture medium (Procell Life Science\&Technology). Both cell lines were maintained at $37^{\circ} \mathrm{C}$ in a humidified atmosphere containing $5 \% \mathrm{CO} 2$.

Get rid of the culture medium from the petri dish and wash it three times with phosphate-buffered saline (PBS). Cells were collected by scraping off after the addition of cell lysis buffer (Beyotime, Shanghai), and then mixed and place at a standstill for 30 minutes. Extract supernatant after centrifugation to collect cell Sample.

\section{Quantitative reverse transcription- polymerase chain reaction (qRT-PCR)}

Total RNA of all samples in our experiment was extracted using the M5 Universal RNA Mini Kit (Mei5bio, Beijing). Cells were collected by scraping with phosphate-buffered saline (PBS) and extracting the supernatant after mixing well with $500 \mu$ lysis solution. The supernatant was added with an equal volume of $70 \%$ ethanol and transferred to an adsorption column for centrifugation. Add protein solution RW and repeat centrifugation. Next, add rinse solution to the adsorption column and centrifuge (twice). Lastly, Add $50 \mu \mathrm{l}$ RNase-FreeH2O to the adsorbed membrane according to the expected RNA yield. The measured RNA concentration is $429 \mathrm{ng} / \mu \mathrm{l}$ (MCF-7) and $459 \mathrm{ng} / \mu \mathrm{l}(10 \mathrm{~A})$. Then, we conducted reverse transcription assays using the M5 Sprint qPCR RT kit with gDNA remover (Mei5bio, Beijing). The total volume of the CDNA reaction system was $10 \mu \mathrm{l}$. The quantitative polymerase chain reaction (q-PCR) analysis was performed using CFX Connect Real-Time System (Bio-Rad, USA). Pre-denaturation was performed at $95^{\circ} \mathrm{C}$ for $30 \mathrm{~s}$ and cycled once. PCR reaction was performed at $95^{\circ} \mathrm{C}$ for $5 \mathrm{~s}$ and $60^{\circ} \mathrm{C}$ for 20 s, cycled 40 times. Fusion curve analysis at $95^{\circ} \mathrm{C}$ for $0 \mathrm{~s}$ and $65^{\circ} \mathrm{C}$ for $15 \mathrm{~s}$. The following primer sequences for this assay were used: Armadillo repeat-containing 1 (ARMC1): (forward): $5^{\prime}$-AACTACAAACAAACGTGCCAAAA$3^{\prime} \rrbracket$ (reverse): 5'-ACACACCTTTGAACACACCTATT-3'; Glyceraldehyde-3-phosphate dehydrogenase (GAPDH): (forward): 5'-GGACACTGAGCAAGAGAGGC-3' $\otimes$ (reverse): 5'- TTATGGGGGTCTGGGATGGAA-3'. All the primer sequences came from Tsingke Biotechnology (Beijing, China).

\section{Enzyme-linked immunosorbent assay}

This experiment was completed by using the ARMC1 enzyme-linked immunosorbent assay kit (Jiangsu jingmei Biological Technology, China). Dilute the standard according to concentration size order, and then incubate the test plate for 30 minutes at $37^{\circ} \mathrm{C}$ after adding the standard, sample and distilled water to 
programmed standard holes, sample holes and blank. Put the prepared cleaning solution in each hole and shake it for washing the plate, and then tap the plate gently to get rid of the cleaning solution. Repeat this clean step after the addition of the Enzyme-labeled reagent except for the blank. The developing color solution was added to each hole and placed statically at $37^{\circ} \mathrm{C}$ for 15 minutes in the dark. Lastly, read the results after the addition of the stop solution. Prime 8 software was used to analyze the results of ELISA.

\section{Immunohistochemistry (IHC) analysis}

This experiment was performed by Mouse/rabbit streptavidin-biotin assay system (ZSGB-BIO, Beijing). IHC assays were performed using formalin-fixed BRCA tissue samples which were embedded in $4-\mu \mathrm{m}$ thick paraffin sections. Paraffin sections were deparaffinized by graded alcohol and then incubated by Endogenous Peroxidase Blockers for 20 minutes. The sections were incubated with antibodies against ARMC1 (Novus Biologicals, USA) overnight at $4^{\circ} \mathrm{C}$ after Goat Serum Blocking Solution incubation. The incubation by the secondary antibody for 15 minutes at room temperature, and combined with horseradish enzyme-labeled streptavidin for 10 minutes. Image-Pro Plus 6.0 software was used to perform semiquantitative scoring of the images.

\section{Immunofluorescence staining}

Tissue sections were deparaffinized by graded alcohol and then washed three times with phosphatebuffered saline (PBS), permeabilized with $0.4 \%$ Triton X-100 for 30 min, and blocked with goat serum working liquid (Wuhan Boster Biological Technology, Wuhan, China) for 2 hours after antigen retrieval. The sections were then incubated overnight with mixed primary antibodies at $4^{\circ} \mathrm{C}$, washed in PBS to remove unbound primary antibodies, and incubated with secondary antibodies in the dark at room temperature (RT) for 1 hour. The sections were counterstained with 4', 6 diamidino-2-phenylindole (SigmaAldrich) for 5 minutes and washed with PBS. The primary antibodies included mouse anti-ARMC1 (1:50; Novus Biologicals, USA), outer mitochondrial membrane marker-rabbit anti-TOM20 (the outer mitochondrial membrane 20) antibody (1:200; Wuhan Proteintech, Wuhan, China), and inner mitochondrial membrane marker-rabbit anti-TIM23 (the inner mitochondrial membrane 23) antibody (1:200; Wuhan Proteintech, Wuhan, China). The fluorophore-conjugated secondary antibodies used were goat anti-rabbit Alexa Fluor 488 (1:500; Abbkine, Wuhan, China), and goat anti-mouse Alexa Fluor 549 (1:500; Abbkine, Wuhan, China). Images were captured by confocal laser scanning microscopy (Nikon $A 1+R$, Japan). The fluorescence intensity was analyzed by using Image-J software.

\section{Quantification of Staining Expression}

The image-pro plus (Version 6.0.0.260) was used to evaluate the ArmC1 expression in immunohistochemistry. Measure the $\mathrm{AOI}$ (area of interest) including average radius, perimeter, and optical density of staining position. After statistics, The IOD (integrated optical density) was calculated by 
density(mean)『Area density. The expression level is judged from the ratio of SUM value of IOD to SUM value of Area.

\section{Statistical analysis}

Receiver operating characteristic (ROC) curve analysis was used to settle the optimal cutoff value of the ARMC1. The optimal cutoff value of the ARMC1 was determined by the maximization of the Youden's index [Sensitivity-(1-Specificity)]. Relations of the ARMC1 to other variables were evaluated using the $\chi^{2}$ test or Spearman's rank correlation coefficient. All statistical analyses were performed using SPSS version 23.0 software. $p<0.05$ was considered to indicate a significant difference.

\section{Result:}

\section{The expression profiles of ARMC1 in BRCA samples}

The expression profiles from the GEPIA database showed that ARMC1 expression presents a higher level in breast tumor samples than in normal samples $(T=1085, N=291)$ (Figure 1A). Also, higher expression of ARMC1 was significantly associated with a lower survival rate ( $p \bigotimes 0.05)$ (Figure 1B).

By exploring the ARMC1 expression profiles in the HPA database, it could be seen that ARMC1 mRNA expression in normal breast tissue was lower than that in other tissues (Figure 1C). However, we found the ARMC1 mRNA level relatively increased in BCRA samples compared with other types of carcinoma (Figure 1D). Besides, ARMC1 protein significantly presents higher expression in breast tissue than other tissues (Figure 1E, F).

\section{Prognostic evaluation of ARMC1 level in BRCA patients}

The Kaplan-Meier plotter tool was used to detect the correlation between ARMC1 level and overall survival in BRCA. The result suggests that the ARMC1 level was negatively associated with prognostic survival (Figure 2A, Hazard Ratio (HR)=1.37, 95\% confidence interval (Cl):1.23-1.51, $p=1.4 \mathrm{E}-09$ ).

Immunohistochemistry (IHC) images from the HPA database showed that the intensity of tumor samples staining was stronger than that in normal samples (Figure 2B). Next, the survival analysis in the HPA database confirmed the results that increased ARMC1 level is associated with poor survival (Figure 2C).

Next, we explored the risk factors associated with ARMC1 expression in TCGA-BRCA patients. The TCGABRCA clinical parameters were collected from the HPA database. Fragments Per Kilobase per Million (FPKM) was used to measure ARMC1 genetic expression. We included 869 patients with seven clinical parameters for this analysis. The average age of the patients was 58 (interquartile range: 48-67). As shown in Table 1, the tumor site was left in 443 cases and right in 419 cases. There were 608 cases of ductal carcinoma, 215 cases of lobular carcinoma, and 45 cases of other types. The majority of cases 
(498 cases) presented with stage II carcinoma. Fewer were of stage III (189 cases) and stage I (156 cases). Lymphovascular invasion was present in 442 patients. 408 patients had node-positive disease. In the pathological T stage, the majority (493) of cases were stage T2. The rest presented were 240 cases (T1) and 98 cases (T3). In the pathological N stage, the majority (408) of cases were stage N0, and the rest presented 296 cases of stage N1, 88 cases of stage N2, and 58 cases of stage N3. $\chi^{2}$ test showed tumor stage ( $p \otimes 0.001)$, Histology ( $p \otimes 0.001)$, pathological T stage $(p \otimes 0.001)$, and pathological $N$ stage ( $p \square$ 0.001) were associated with AMRC1 expression (Supplement Table 2). ROC analysis identified 16.05 as the best cutoff value of ARMC1 by the Youden's index [Sensitivity-(1-Specificity)] (Table 2). Curve Area as shown in Supplement Figure1.

\section{Table 1}

\section{Clinicopathological characteristics of BRCA samples}




\begin{tabular}{|c|c|}
\hline & NO.(\%) \\
\hline \multicolumn{2}{|l|}{ Location } \\
\hline Left site & $443(51)$ \\
\hline Right site & $419(48.2)$ \\
\hline Unknown & $7(0.8)$ \\
\hline \multicolumn{2}{|l|}{ Histology } \\
\hline Ductal carcinoma & $608(70)$ \\
\hline Lobular carcinoma & $215(24.7)$ \\
\hline Other types of carcinoma & $45(5.2)$ \\
\hline Unknown & $1(0.1)$ \\
\hline \multicolumn{2}{|l|}{ Nathologic tumor stage } \\
\hline Stage $\otimes$ & 156(18) \\
\hline 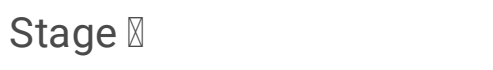 & 498(57.3) \\
\hline 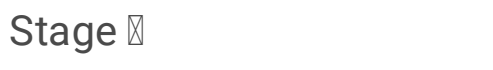 & $189(21.7)$ \\
\hline Stage $\mathbb{Z}$ & $12(1.4)$ \\
\hline Unknown & $14(1.6)$ \\
\hline \multicolumn{2}{|l|}{ Nodal status } \\
\hline $\mathrm{N}+$ & $442(50.9)$ \\
\hline N- & $408(47)$ \\
\hline Unknown & $19(2.1)$ \\
\hline \multicolumn{2}{|l|}{ Nodal status, nr. of + } \\
\hline 0 & $367(42.2)$ \\
\hline $1-3$ & $249(28.6)$ \\
\hline $4-9$ & $85(9.8)$ \\
\hline$\geq 10$ & $50(5.8)$ \\
\hline Unknown & 118(13.6) \\
\hline \multicolumn{2}{|l|}{ Lymphovascular invasion } \\
\hline Present & $442(50.9)$ \\
\hline Absent & $408(47)$ \\
\hline Unknown & $19(2.1)$ \\
\hline
\end{tabular}




\begin{tabular}{|ll|}
\hline T stage & \\
\hline T1 & $240(27.6)$ \\
T2 & $493(56.7)$ \\
T3 & $98(11.3)$ \\
T4 & $28(3.2)$ \\
Unknown & $10(1.2)$ \\
\hline N stage & \\
\hline N0 & $408(46.9)$ \\
N1 & $296(34.1)$ \\
N2 & $88(10.1)$ \\
N3 & $58(6.7)$ \\
Unknown & $19(2.2)$ \\
\hline
\end{tabular}

Table2

ROC curve parameter considering the most discriminatory cutoff value of ARMC1

\begin{tabular}{|c|c|c|c|c|c|c|c|}
\hline Parameter & AUC & $95 \% \mathrm{Cl}$ & Sensitivity & Specificity & $\begin{array}{l}\text { Cut off } \\
\text { value }\end{array}$ & $\begin{array}{l}\text { Youden } \\
\text { index }\end{array}$ & $\begin{array}{l}\text { P- } \\
\text { value }\end{array}$ \\
\hline FPKM & 0.803 & $\begin{array}{l}0.753- \\
0.853\end{array}$ & 0.761 & 0.293 & 16.05 & 0.467 & $\otimes 0.001$ \\
\hline \multicolumn{8}{|c|}{$\begin{array}{l}\text { AUC, Area under the ROC curve; } \mathrm{Cl} \text {, confidence interval; } \mathrm{ROC} \text {, receiver operating characteristic } \\
\text { curve; ARMC1, Armadillo repeat-containing 1; }\end{array}$} \\
\hline \multicolumn{8}{|c|}{ *Statistically significant $(p<0.05)$} \\
\hline
\end{tabular}

\section{The Genomic mutations of ARMC1 and its biological pathways in BRCA}

The data from the cBioPortal database indicates that ARMC1 was altered in 332 of 994 samples (32\%). 289 (90\%) high expression samples contained 45 amplified samples and 1 fusion mutation, 59 (18.3\%) ARMC1 amplified samples had 45 high expressions and 1 low expressed sample, 2 (1\%) truncating mutation, and $2(1 \%)$ fusion mutation (Figure 3A). As shown in Figure 3B, breast invasive ductal carcinoma was majorly associated with ARMC1 mutations, and the types of mutations were mainly the mRNA high expression and amplification. Besides, lower survival was significantly presented in the 
mutated samples group than that in the group without ARMC1 mutation (Figure 3C, Altered group=322 unaltered group $=672 p=0.0203$ ).

Neighboring genes related to ARMC1 mutation were found in the cBioPortal database, TP53 $(51.24 \%)$, TRPS1 (31.99\%), and RAD21 (30.75\%) were identified as the top three mutant genes (Figure 3D). Next, we used the neighboring genes associated with ARMC1 mutations to analyze the biological networks by the DAVID Functional Annotation Tool (Figure 3E). In the cellular component, the endoplasmic reticulum membrane and nucleus were significantly related to ARMC1 mutations. These mutations also affect molecular functions including the activity of various important ion channel modulators such as chloride, calcium, and sodium in particular. Mitochondrial permeability regulation and cell death were also identified be involved in these mutations. In biological processes, some vital functions include positive regulation of transporter activity, chromosome organization, sodium ion transportation, homeostasis, and regulating DNA binding transcription factor activity that was all significantly related to ARMC1 mutations.

\section{Enrichment analysis of co-expressed genes relating to ARMC1 in BRCA.}

The cBioPortal database was used to discover the co-expressed genes positively or negatively associated with ARMC1 in BRCA. As showed in Figure 4A, most of these genes were positively correlated with ARMC1. After finding out the co-expressed genes associated with ARMC1, we analyzed the biological functions of these differential genes by Gene set enrichment analysis (GESA). Interestingly, these differential genes were mainly located in the nucleoplasm. Protein binding and poly (A) RNA binding were their primary molecular functions. More importantly, biological processes involved in these differential genes include regulating cell cycle and positive regulation of transcription from RNA polymerase $\square$ promoter, Etc. (Figure 4B-D). The KEGG analysis also showed the potential effect of these differential genes in the cell cycle (Figure 4E).

\section{ARMC1 was highly expressed in breast tumor tissues and was mainly located in the mitochondrial membrane}

The qRT-PCR assay showed that ARMC1 mRNA level was highly expressed in MCF-7 cell lines compared with the MCF-10A cell lines (Figure 5A, $p<0.01$ ). For further quantitative analysis of ARMC1 expression, we performed the enzyme-linked immunosorbent assay in two types of cell lines, the results showed that ARMC1 expression was higher in MCF-7 cell lines than that in the MCF-10A cell lines (Figure 5B, $p<0.01$ ). Besides, it was obviously found in the immunohistochemistry that ARMC1 was higher expressed in BRCA tissues than that in the para-carcinoma tissue (Figure $5 \mathrm{C}, p<0.01$ ). According to the previous studies that ARMC1 is mainly localized to the mitochondrial. It could be seen in the results of immunofluorescence that ARMC1 was localized at the both inner mitochondrial membrane and outer mitochondrial membrane in invasive ductal breast carcinoma and lobular breast carcinoma (Figure 5D-E). 


\section{Discussion:}

Invasive breast carcinoma (BRCA) is one of the most common diseases in female patients, and various targeted treatments have been applied to BRCA. However, the heterogeneity and wide invasion make BRCA difficult to be cured completely [20]. This study primarily reveals the role of ARMC1 as a novel biomarker in invasive breast cancer and provides a significant regulatory target for the diagnosis and treatment of BRCA.

Armadillo (Arm) repeat proteins are initially found in drosophila segment polarity protein as multifunctional protein families [21]. The tandem Arm repeat structure consisting of 42 repetitive amino acids enables Arm repeat proteins to get involved in intracellular signaling and cytoskeletal regulation [22]. After binding the Wnt signal through the Arm repeat region, the oncogenic molecule $\beta$-catenin can bind the transcription factors of the T-Cell Factor (TCF) family, and then induce the cell proliferation genes expression $[23,24]$. Numerous studies have examined the association of the Arm repeat protein family with tumor development. However, the role of ARMC1 in BRCA remains unclear. In this study, we examined the ARMC1 expression in both normal samples and tumor samples by the HPA database, and the result indicates that ARMC1 mRNA level was lowly expressed in normal samples but highly expressed in tumor samples. This differential expression highlights the significance of AMRC1 in BRCA.

After we learned about the high expression of ARMC1 in invasive breast carcinoma, we set out to explore its prognostic value by the TCGA-BRCA cohort. The Kaplan-Meier analysis indicates that increased expression of ARMC1 was significantly associated with poor survival, which we also verified from the TCGA-BRCA cohort. It could be found that factors such as tumor pathological grade and histology were significantly associated with the prognosis of BRCA by $\chi^{2}$ test of 869 clinical patient information in the TCGA database.

Numerous analyses of cancer genomes prove that tumor development is usually accompanied by genetic mutations [25]. The genetic mutations are also recognized as 'drivers' for tumorigenesis because of their ability to induce abnormal cell proliferation [26]. Therefore, we tried to explore the carcinogenesis mechanism of ARMC1 in BRCA in terms of gene mutation. The cBio Cancer Genomics Portal database data indicates that $32 \%$ of gene mutations were found in BRCA, and amplification and high transcription account for most of these mutations. The genetic mutations of ARMC1 were also significantly related to poor survival. Especially, TP53 is one of the highly correlated genes in ARMC1 mutations. Its frequent mutations of exon 4 and intron 3 were reported in several breast cancer studies that can directly or indirectly cause DNA repair disorders, abnormal cell cycles [27]. Besides, the networks of biological functions show that ARMC1 and neighboring genes were involved in the positive regulation of transporter activity and regulation of various essential ion channels. It is reported that calcium-activated chloride channels activate EGFR/STAT3 signaling, which can lead to abnormal proliferation of breast cancer cells [28]. This abnormal ion channel activity caused by ARMC1 mutation may be involved in this signal transduction. 
At last, we found the adjacent co-expressed genes associated with ARMC1 and then performed the genes sets enrichment analysis to explore the possible regulatory mechanism of ARMC1 in BRCA. The functional networks of GO analysis showed that nucleoplasm is majorly associate with ARMC1. The primary molecular function is protein binding. More importantly, KEGG analysis showed that coexpressed genes of ARMC1 are involved in the cell cycle. The eventual result of cell cycle dysregulation is abnormal cell proliferation [29]. The genes associated with cell cycle regulation such as PRKDC and E2F transcription factor 5 (E2F5) was found in our study. It has been reported that the overexpression of PRKDC in breast cancer leads to cell proliferation by accelerating the G2/M cell cycle [30]. In addition, aberrant overexpression of E2F5 in breast cancer is also related to cell proliferation [31]. There are many co-expressed genes associated with ARMC1 that were identified to be involved in cell cycle regulation. ARMC1 may be directly or indirectly involved in breast carcinogenesis by affecting these regulators associated with cell proliferation. More studies are required to prove the relationship between ARMC1 and these co-expressed genes in BRCA.

Numerous experiments have demonstrated the differential expression of ARMC1 in BRCA, which provides concrete evidence that ARMC1 works as a crucial target in BRCA development. Meanwhile, this differential expression can serve as a starting point for further study of the ARMC1 mechanism in BRCA. Mitochondria serve as a regulatory center for cellular metabolism, its dysfunction can lead to activation of potential oncogenic pathways [32]. Recently, an increasing number of studies have found that mitochondria can be used as an effective target in breast cancer therapy [33]. It is well-known that mitochondria are an important hub for various ion transport and signal transduction, and ion channels and transporters on mitochondrial bilayer membranes maintain normal cellular homeostasis [34, 35]. There are various ion channels located in the outer or inner mitochondrial membrane that were identified as associated with cancer development. For instance, the overexpression of voltage-dependent anion channels 1 (VDAC1) has been found in cancer cells, it increases the glycolytic rate by the direct mitochondrial ATP transport. This abnormal glucose metabolism promotes the proliferation and migration of tumor cells (the Warburg effect) [36]. Surprisingly, we found that ARMC1 is mainly localized on both the inner and outer mitochondrial membranes in the tissues of the ductal and lobular breast carcinoma. Besides, the ARMC1 mutations may affect various ion channel activities including calcium ions in our result. All the detail revealed that ARMC1 may affect BRCA development by mitochondrial ionic homeostasis. Although ARMC1 acts as a molecule on the mitochondrial membrane, the mechanism of ARMC1 in BRCA remains unknown. Therefore, we expect to find more evidence to identify the role of ARMC1 in BRCA.

\section{Conclusion:}

Our results suggest that ARMC1 could be a potential biomarker in invasive breast cancer, it provides valuable clues for the treatment and diagnosis of invasive breast cancer. However, this experiment has some limits. Reliable prognostic model analysis cannot be performed due to the limited information of the TCGA-BRCA patient cohort. Thus, more attempts are needed to demonstrate the effect of ARMC1 on the prognosis of BRCA from other pathways. 


\section{Abbreviations}

BRCA: Invasive breast carcinoma; ARMC1: Armadillo repeat-containing 1; GO: gene ontology ; KEGG: Kyoto Encyclopedia of Genes and Genomes; ARMC12: Armadillo repeat-containing 12; APC: Adenoma polyposis coli; GSK3ß: glycogen synthase kinase 3ß; GEPIA: Gene Expression Profiling Interactive Analysis; GTEx: Genotype-Tissue Expression; HPA: Human Protein Atlas; TCGA: The Cancer Genome Atlas; MCF-7: Human breast carcinoma cell lines; MCF-10A: Human normal breast cells; DMEM: dulbecco's modified eagle medium; FBS: fetal bovine serum; PBS: phosphate-buffered saline; AOI: Area of interest; IOD: Integrated optical density; ELISA: Enzyme-linked immunosorbent assay; IHC: Immunohistochemistry; ROC: Receiver operator characteris-tic curve; mRNA: messenger RNA; HR: hazard ratio; Cl: confidence interval; FPKM: fragments per kilobase per million; TRPS1: tricho-rhino-phalangeal syndrome 1; GESA: gene set enrichment analysis; Arm: Armadillo; EGFR: epidermal growth factor receptor; STAT3: signal transducer and activator of transcription 3; PRKDC: protein kinase, DNA-activated, catalytic subunit; E2F5: E2F transcription factor 5; VDAC1: voltage-dependent anion channels 1; ATP: Adenosine triphosphate; RT: Room temperature.

\section{Declarations}

\section{Acknowledgments:}

Not applicable.

\section{Authors' contributions:}

YG and FZ contributed equally to this article. YG performed the cell culture as well as the collection and analysis of the data, and was a major contributor in writing the manuscript. FZ carried out the operation of tissue collection and related experiments. HW and LL designed the study and revised the manuscript. All authors read and approved the final manuscript.

\section{Funding:}

The author would like to thank the Pathological Diagnosis Center of Chongqing Medical University for assistance with clinical samples. This study was supported by grants from the Chongqing Natural Science Foundation (cstc2020jcyj-msxmX0144).

\section{Availability of data and materials $\llbracket$}

The datasets analysed or generated during the current study are available in the Gene Expression Profiling Interactive Analysis (http://gepia.cancer-pku.cn/), cBio Cancer Genomics Portal repository (http://cbioportal.org) Kaplan-Meier plotter, (http://kmplot.com/analysis/) Human 


\section{Ethics approval and consent to participate区}

The Ethics Committee approved the study of the Chongqing Medical University, and all subjects obtained informed consent. All procedures performed in research involving human subjects were in accordance with the ethical standards of the institutional and/or national research committees, the 1964 Declaration of Helsinki and its subsequent amendments, or similar ethical standards.

\section{Consent for publication】}

Not applicable.

\section{Competing interests:}

The authors declare that they have no competing interests.

\section{Author details:}

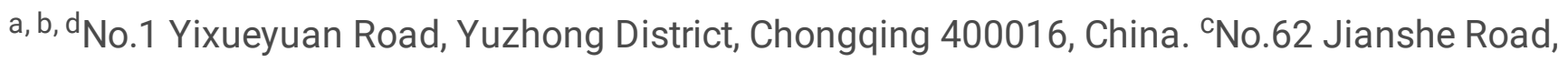
Shuanglonghu Street, Yubei District, Chongqing 400016, China.

\section{References}

1. Vieira, R.A., A.M. da Costa, J.L. de Souza, R.R. Coelho, C.Z. de Oliveira, A.J. Sarri, et al., Risk Factors for Arm Lymphedema in a Cohort of Breast Cancer Patients Followed up for 10 Years. Breast Care (Basel), 2016. 11(1): p. 45-50.

2. Torre, L.A., F. Bray, R.L. Siegel, J. Ferlay, J. Lortet-Tieulent, and A. Jemal, Global cancer statistics, 2012. CA Cancer J Clin, 2015. 65(2): p. 87-108.

3. Jafari, S.H., Z. Saadatpour, A. Salmaninejad, F. Momeni, M. Mokhtari, J.S. Nahand, et al., Breast cancer diagnosis: Imaging techniques and biochemical markers. J Cell Physiol, 2018. 233(7):

p. 5200-5213.

4. Peart, O., Breast intervention and breast cancer treatment options. Radiol Technol, 2015. 86(5): p. 535M-558M; quiz 559-62.

5. DeSantis, C.E., J. Ma, A. Goding Sauer, L.A. Newman, and A. Jemal, Breast cancer statistics, 2017, racial disparity in mortality by state. CA Cancer J Clin, 2017. 67(6): p. 439-448.

6. Watkins, E.J., Overview of breast cancer. Jaapa, 2019. 32(10): p. 13-17. 
7. Tewari, R., E. Bailes, K.A. Bunting, and J.C. Coates, Armadillo-repeat protein functions: questions for little creatures. Trends Cell Biol, 2010. 20(8): p. 470-81.

8. Valkenburg, K.C., C.R. Graveel, C.R. Zylstra-Diegel, Z. Zhong, and B.O. Williams, Wnt/ $\beta$-catenin Signaling in Normal and Cancer Stem Cells. Cancers (Basel), 2011. 3(2): p. 2050-79.

9. Khramtsov, A.I., G.F. Khramtsova, M. Tretiakova, D. Huo, O.I. Olopade, and K.H. Goss, Wnt/betacatenin pathway activation is enriched in basal-like breast cancers and predicts poor outcome. Am J Pathol, 2010. 176(6): p. 2911-20.

10. Li, D., H. Song, H. Mei, E. Fang, X. Wang, F. Yang, et al., Armadillo repeat containing 12 promotes neuroblastoma progression through interaction with retinoblastoma binding protein 4 . Nat Commun, 2018. 9(1): p. 2829.

11. Munemitsu, S., I. Albert, B. Souza, B. Rubinfeld, and P. Polakis, Regulation of intracellular beta-catenin levels by the adenomatous polyposis coli (APC) tumor-suppressor protein. Proc Natl Acad Sci U S A, 1995. 92(7): p. 3046-50.

12. Wagner, F., T.C. Kunz, S.R. Chowdhury, B. Thiede, M. Fraunholz, D. Eger, et al., Armadillo repeatcontaining protein 1 is a dual localization protein associated with mitochondrial intermembrane space bridging complex. PLoS One, 2019. 14(10): p. e0218303.

13. Tang, Z., C. Li, B. Kang, G. Gao, C. Li, and Z. Zhang, GEPIA: a web server for cancer and normal gene expression profiling and interactive analyses. Nucleic Acids Res, 2017. 45(W1): p. W98-w102.

14. Hou, G.X., P. Liu, J. Yang, and S. Wen, Mining expression and prognosis of topoisomerase isoforms in non-small-cell lung cancer by using Oncomine and Kaplan-Meier plotter. PLoS One, 2017. 12(3): p. e0174515.

15. Asplund, A., P.H. Edqvist, J.M. Schwenk, and F. Pontén, Antibodies for profiling the human proteomeThe Human Protein Atlas as a resource for cancer research. Proteomics, 2012. 12(13): p. 2067-77.

16. Tomczak, K., P. Czerwińska, and M. Wiznerowicz, The Cancer Genome Atlas (TCGA): an immeasurable source of knowledge. Contemp Oncol (Pozn), 2015. 19(1a): p. A68-77.

17. Cerami, E., J. Gao, U. Dogrusoz, B.E. Gross, S.O. Sumer, B.A. Aksoy, et al., The cBio cancer genomics portal: an open platform for exploring multidimensional cancer genomics data. Cancer Discov, 2012. 2(5): p. 401-4.

18. Zhou, Y., B. Zhou, L. Pache, M. Chang, A.H. Khodabakhshi, O. Tanaseichuk, et al., Metascape provides a biologist-oriented resource for the analysis of systems-level datasets. Nat Commun, 2019. 10(1): p. 1523.

19. Huang, D.W., B.T. Sherman, Q. Tan, J. Kir, D. Liu, D. Bryant, et al., DAVID Bioinformatics Resources: expanded annotation database and novel algorithms to better extract biology from large gene lists. Nucleic Acids Res, 2007. 35(Web Server issue): p. W169-75.

20. Anastasiadi, Z., G.D. Lianos, E. Ignatiadou, H.V. Harissis, and M. Mitsis, Breast cancer in young women: an overview. Updates Surg, 2017. 69(3): p. 313-317.

21. Peifer, M., S. Berg, and A.B. Reynolds, A repeating amino acid motif shared by proteins with diverse cellular roles. Cell, 1994. 76(5): p. 789-91. 
22. Coates, J.C., Armadillo repeat proteins: beyond the animal kingdom. Trends Cell Biol, 2003. 13(9): p. 463-71.

23. MacDonald, B.T., K. Tamai, and X. He, Wnt/beta-catenin signaling: components, mechanisms, and diseases. Dev Cell, 2009. 17(1): p. 9-26.

24. Xu, W. and D. Kimelman, Mechanistic insights from structural studies of beta-catenin and its binding partners. J Cell Sci, 2007. 120(Pt 19): p. 3337-44.

25. Lawrence, M.S., P. Stojanov, C.H. Mermel, J.T. Robinson, L.A. Garraway, T.R. Golub, et al., Discovery and saturation analysis of cancer genes across 21 tumour types. Nature, 2014. 505(7484): p. 495501.

26. Stratton, M.R., P.J. Campbell, and P.A. Futreal, The cancer genome. Nature, 2009. 458(7239): p. 71924.

27. Kaur, R.P., K. Vasudeva, R. Kumar, and A. Munshi, Role of p53 Gene in Breast Cancer: Focus on Mutation Spectrum and Therapeutic Strategies. Curr Pharm Des, 2018. 24(30): p. 3566-3575.

28. Wang, H., F. Yao, S. Luo, K. Ma, M. Liu, L. Bai, et al., A mutual activation loop between the Ca(2+)activated chloride channel TMEM16A and EGFR/STAT3 signaling promotes breast cancer tumorigenesis. Cancer Lett, 2019. 455: p. 48-59.

29. Phan, T.G. and P.I. Croucher, The dormant cancer cell life cycle. Nat Rev Cancer, 2020. 20(7): p. 398411.

30. Zhang, Y., W.K. Yang, G.M. Wen, H. Tang, C.A. Wu, Y.X. Wu, et al., High expression of PRKDC promotes breast cancer cell growth via 388 MAPK signaling and is associated with poor survival. Mol Genet Genomic Med, 2019. 7(11): p. e908.

31. Inagaki, Y., D. Wu, K. Fujiwara, Y. Ishizuka, A. Oguni, T. Tokunaga, et al., Knockdown of E2F5 induces cell death via the TP53-dependent pathway in breast cancer cells carrying wild-type TP53. Oncol Rep, 2020. 44(5): p. 2241-2252.

32. Porporato, P.E., N. Filigheddu, J.M.B. Pedro, G. Kroemer, and L. Galluzzi, Mitochondrial metabolism and cancer. Cell Res, 2018. 28(3): p. 265-280.

33. Lee, J., A.E. Yesilkanal, J.P. Wynne, C. Frankenberger, J. Liu, J. Yan, et al., Effective breast cancer combination therapy targeting BACH1 and mitochondrial metabolism. Nature, 2019. 568(7751): p. 254-258.

34. O'Rourke, B., Mitochondrial ion channels. Annu Rev Physiol, 2007. 69: p. 19-49.

35. Wang, X., P. An, Z. Gu, Y. Luo, and J. Luo, Mitochondrial Metal Ion Transport in Cell Metabolism and Disease. Int J Mol Sci, 2021. 22(14).

36. Peruzzo, R., L. Biasutto, I. Szabò, and L. Leanza, Impact of intracellular ion channels on cancer development and progression. Eur Biophys J, 2016. 45(7): p. 685-707.

\section{Figures}


A

B $\quad$ D

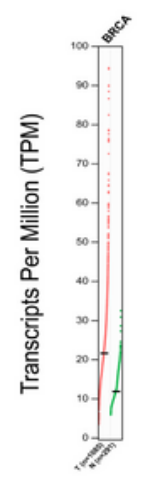

Overall Survival
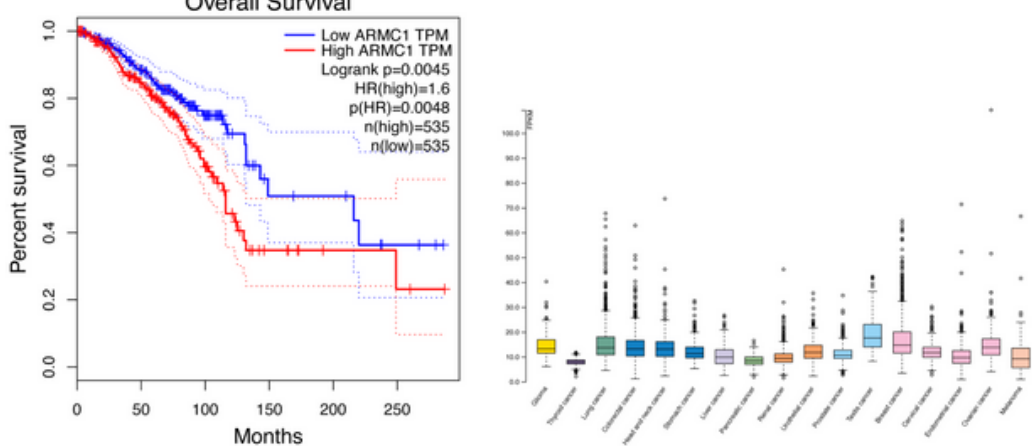

C

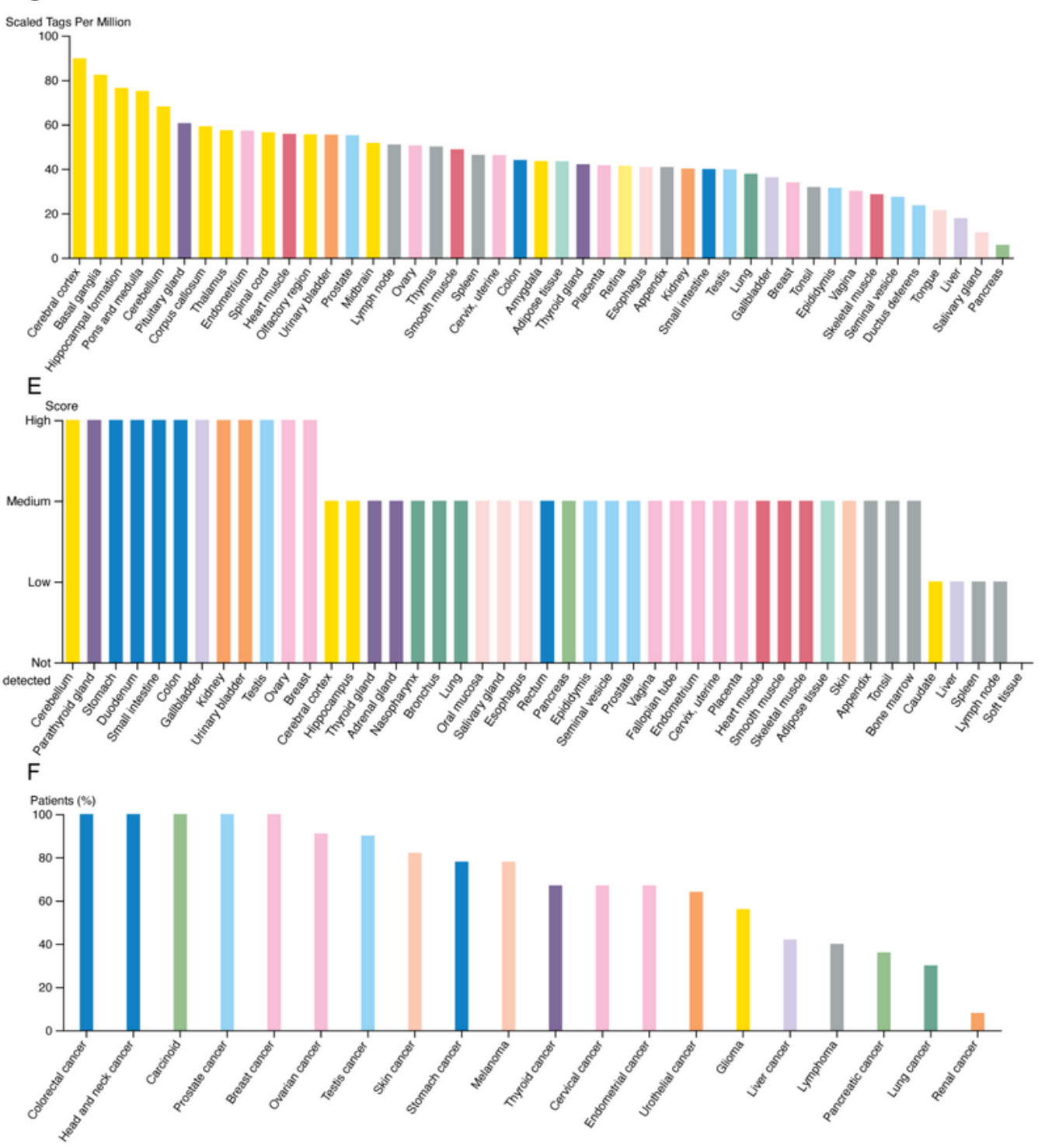

Figure 1

the ARMC1 expression profiles in normal and tumor samples. a The ARMC1 expression profile and boxplot in normal samples and BRCA samples. b The overall survival associated with ARMC1 expression. c The ARMC1 mRNA expression in normal breast tissue. $\mathbf{d}$ The ARMC1 mRNA expression in tumor samples. e The ARMC1 protein expression in normal samples. $\mathbf{f}$ The ARMC1 protein expression in tumor samples. ARMC1, Armadillo repeat-containing 1. 
A

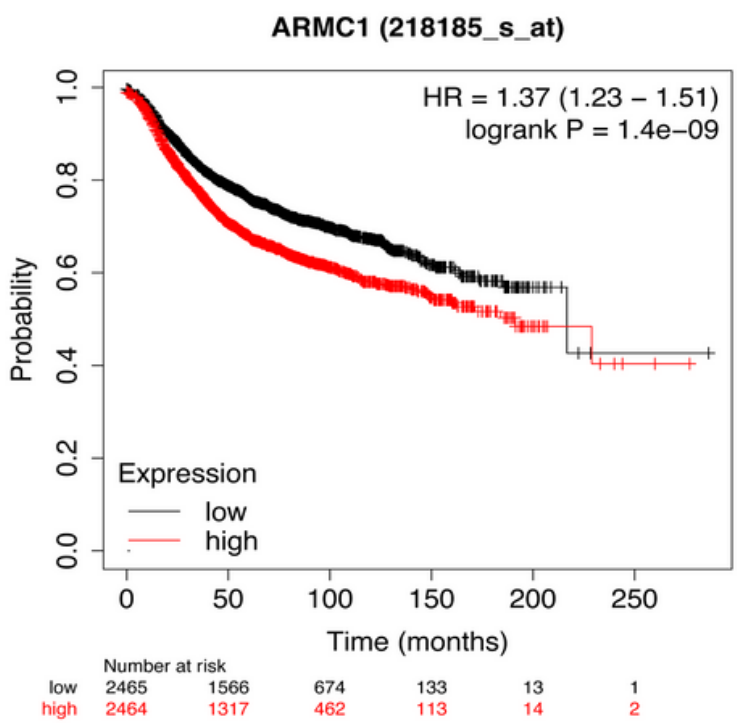

B

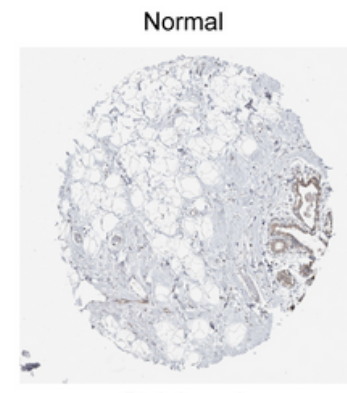

Staining: Low ARMC1 antibody: HPA026085

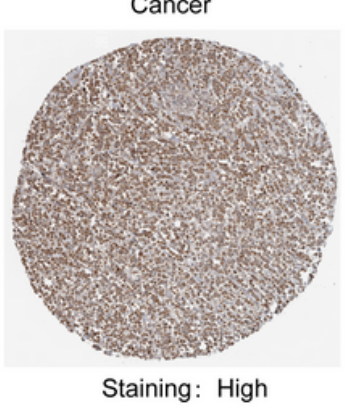

taining: High
C
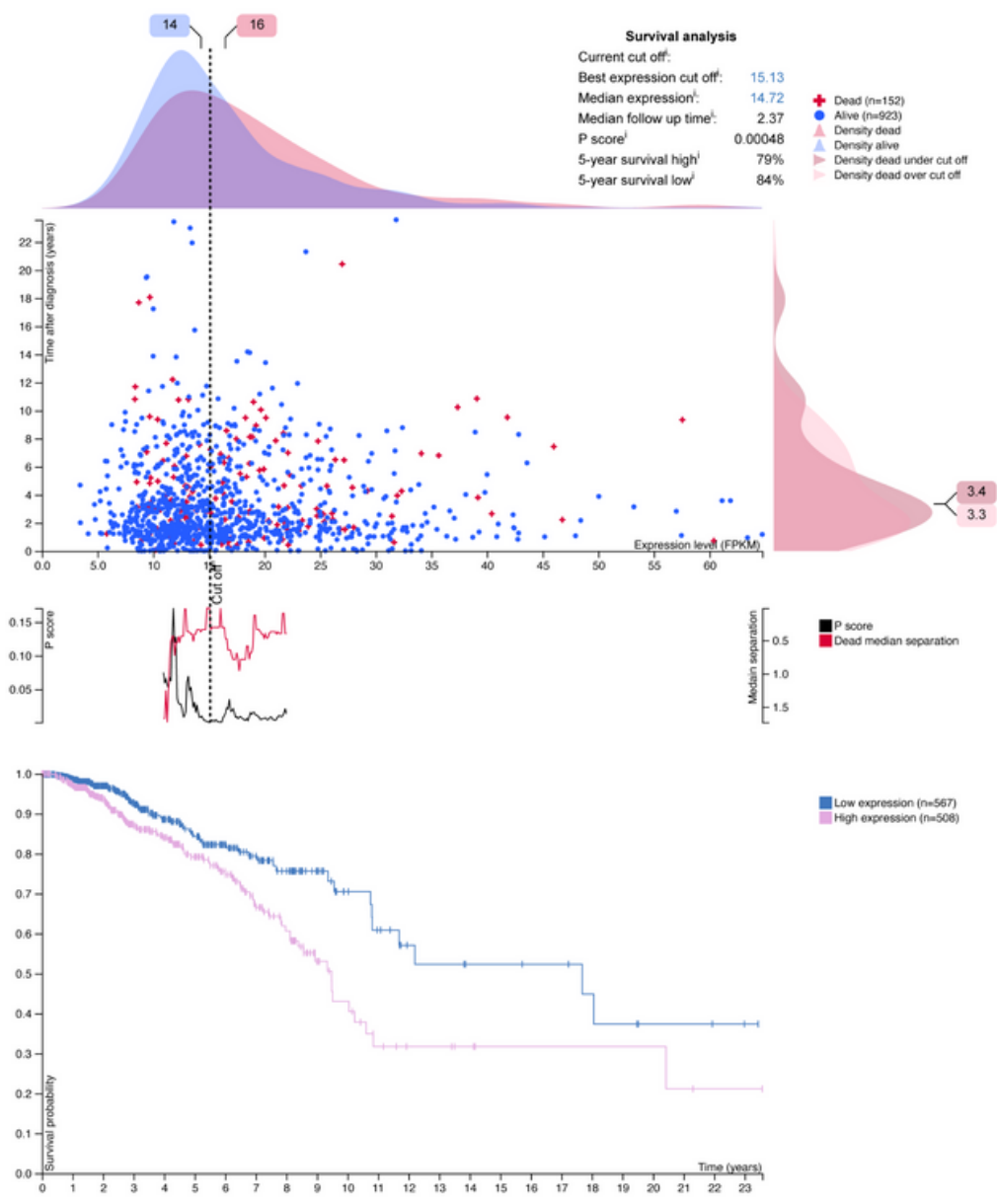

\section{Figure 2}

ARMC1 expression and prognostic evaluation in BRCA samples. a High ARMC1 level was associated with lower survival. b Immunohistochemistry images display that ARMC1 expression in tumor tissue was higher than that in the normal tissue. c Survival analysis in the HPA database showed increased ARMC1 expression associated with poor survival. HR, hazard ratio; ARMC1, Armadillo repeat-containing 1; HPA, Human protein atlas. 
B

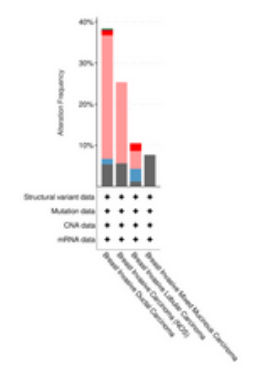

C
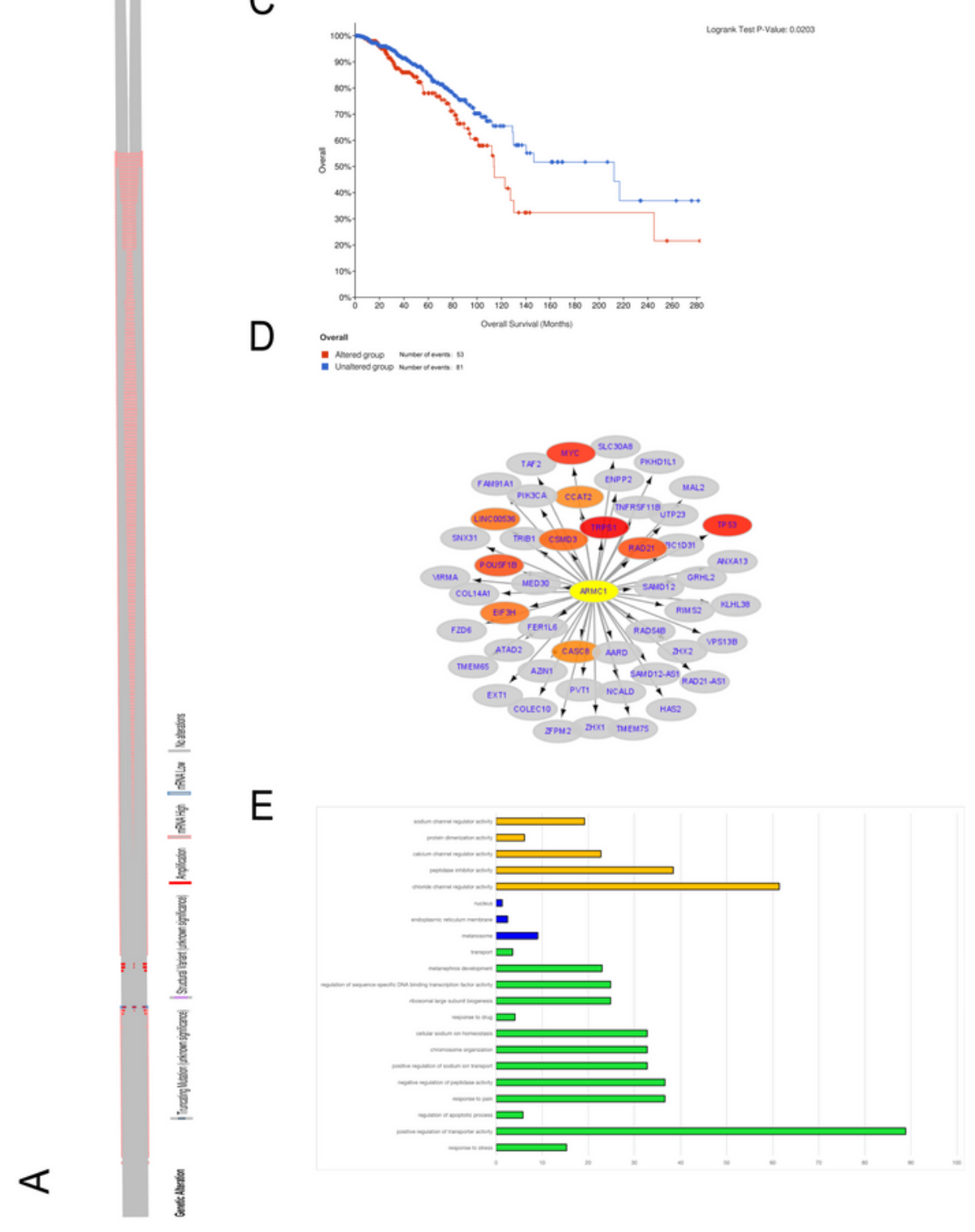

\section{Figure 3}

The mutations of ARMC1 in BRCA and its neighboring genes enriched analysis. a The genetic mutations of ARMC1 in BRCA. $\mathbf{b}$ The cancer types with frequent ARMC1 mutations. $\mathbf{c}$ The overall survival status associated with ARMC1 mutations. $\mathbf{d}$ Neighborhood gene network diagram of ARMC1 mutations. $\mathbf{e}$ Biological function analysis of neighboring genes with ARMC1 mutation. ARMC1, Armadillo repeatcontaining 1; BRCA, Invasive breast carcinoma. 
B
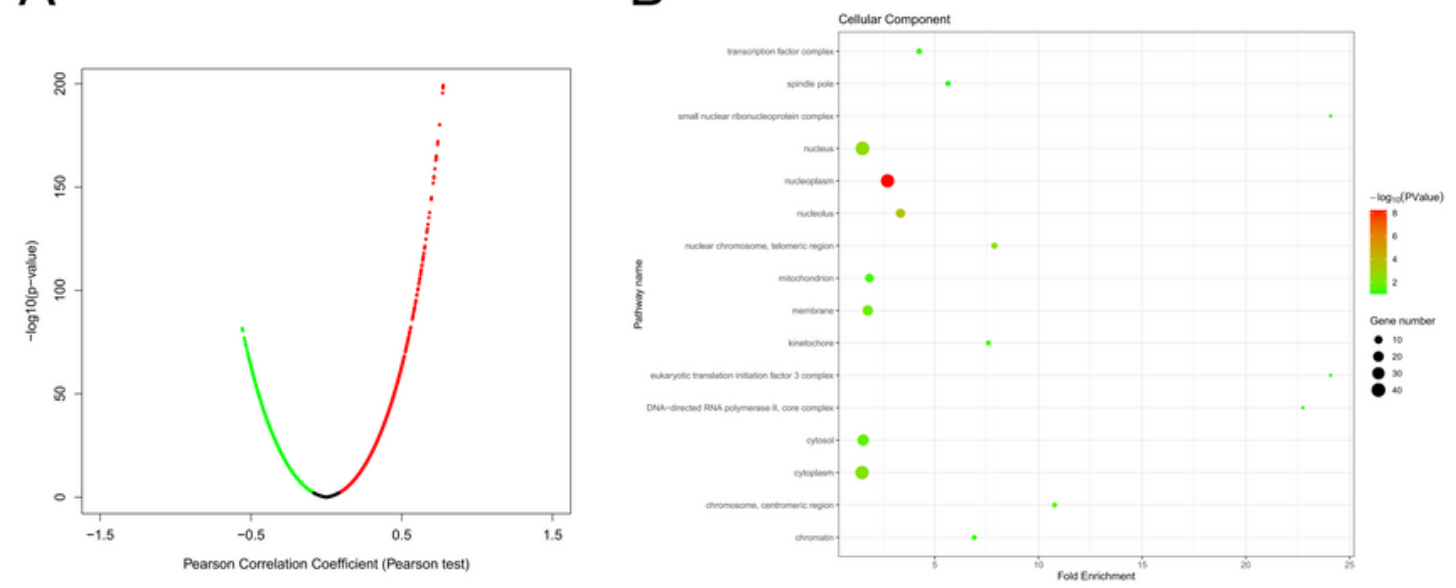

C
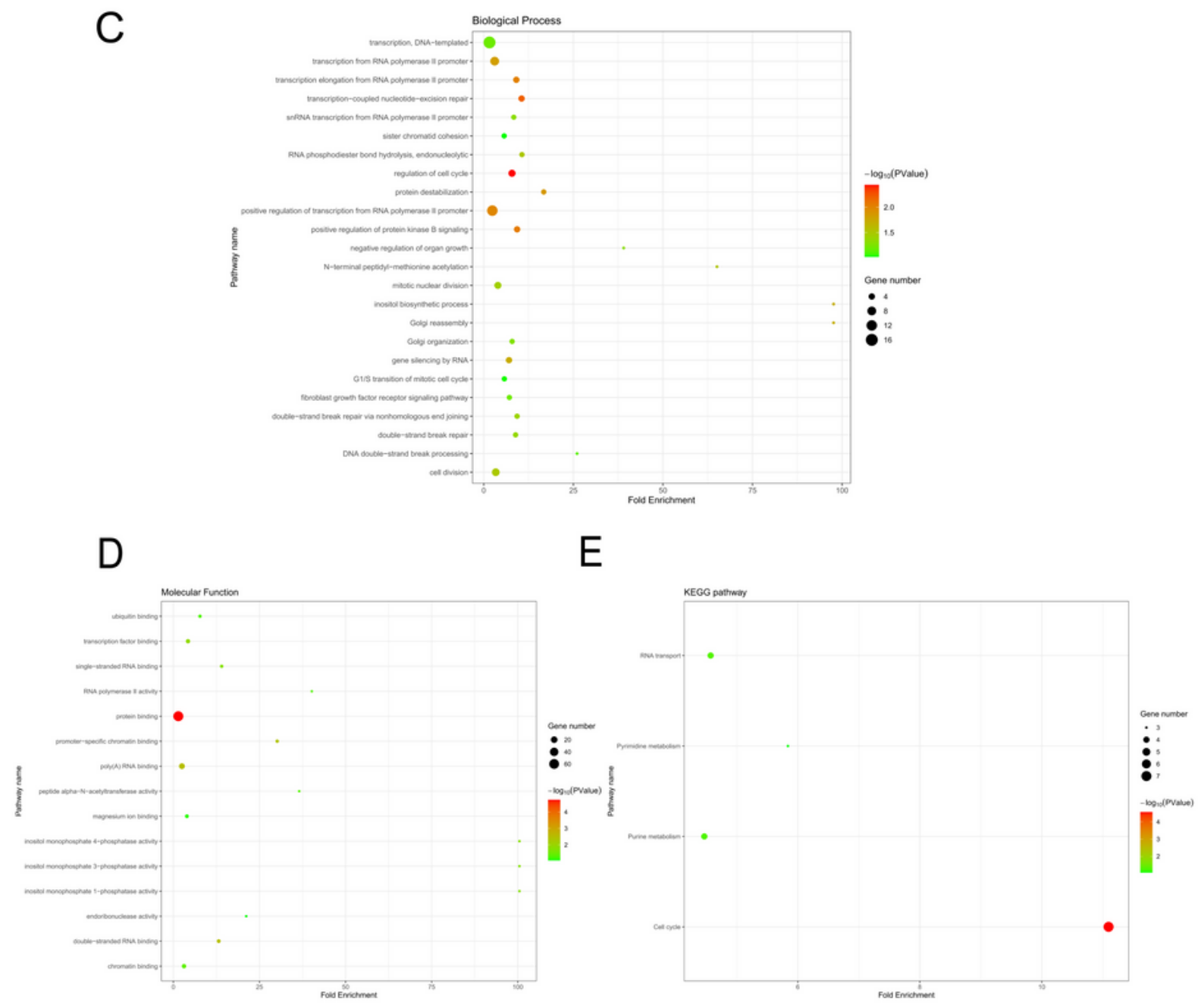

Figure 4

Co-expressed genes with ARMC1 and their enrichment analysis. a Volcano plot showing co-expressed genes associated with ARMC1. b Cellular components. $\mathbf{c}$ Biological processes. $\mathbf{d}$ Molecular functions. $\mathbf{e}$ KEGG pathways. ARMC1, Armadillo repeat-containing 1; KEGG, Kyoto Encyclopedia of Genes and Genomes. 
A

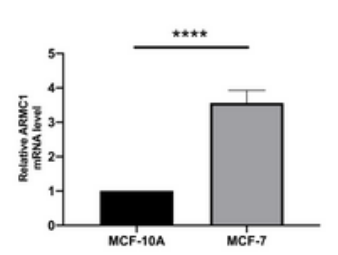

B

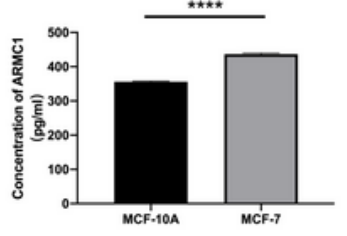

D
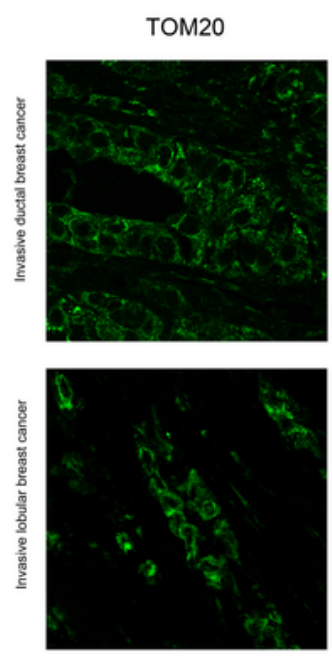

E
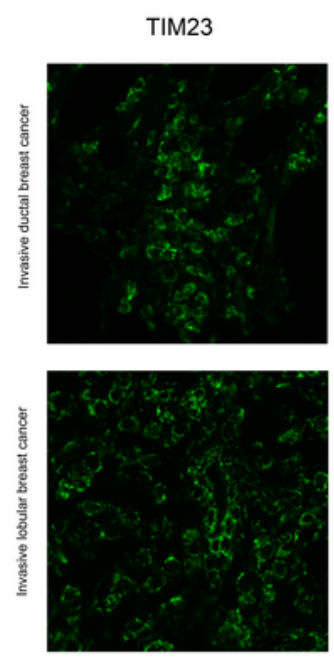

C
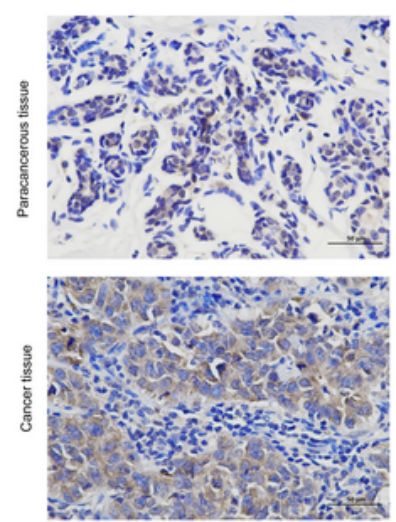

ARMC1
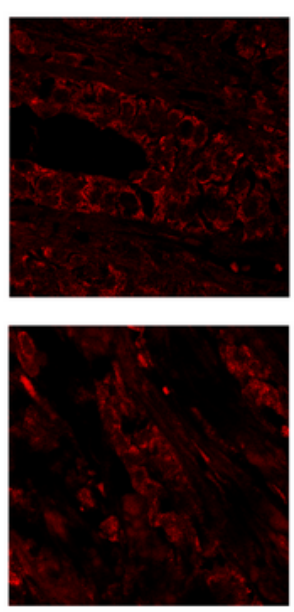

ARMC1
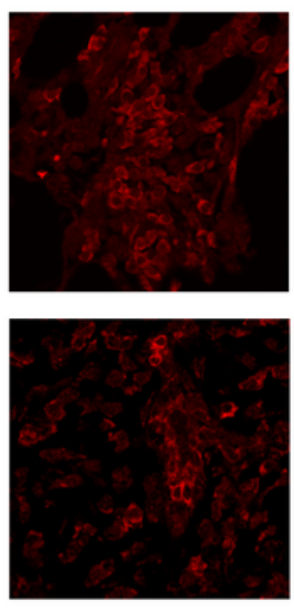

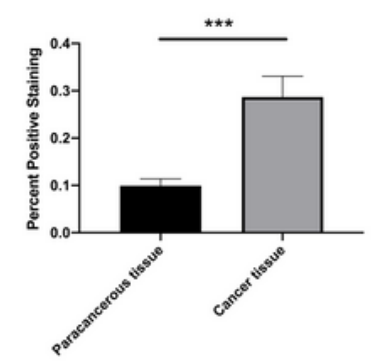

Merge
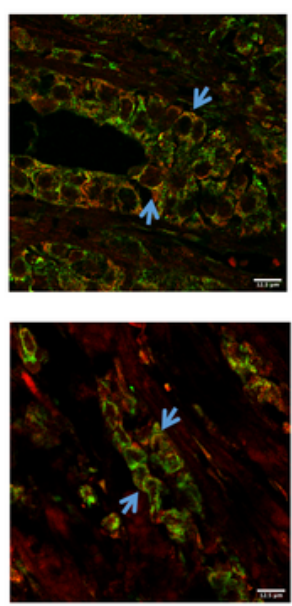

Merge
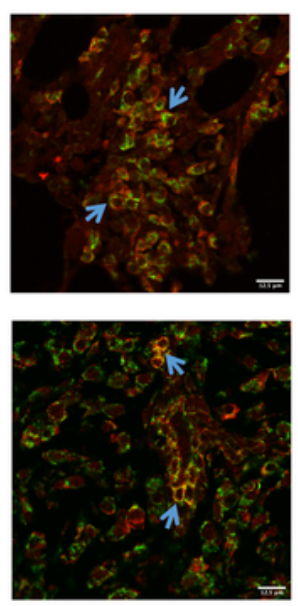

\section{Figure 5}

Validation of ARMC1 expression in BRCA tissues. a ARMC1 mRNA level was higher in MCF-7 cell lines compared with MCF-10A ( $\mathrm{n}=10$ in each group; ${ }^{* \star * *} p<0.0001$ versus controls, Student's t test). b ARMC1 was higher expressed in MCF-7 cell lines compared with MCF-10A ( $\mathrm{n}=15$ in each group; ${ }^{* \star \star \star} p<0.0001$ versus controls, Student's t test). c ARMC1 was higher expressed in BRCA tissues than that in paracarcinoma tissues ( $n=10$ in each group; ${ }^{* * *} p<0.001$ versus controls, Student's t test). Scale bar: $50 \mu m$. d 
ARMC1 was colocalizes with TOM20 in BRCA tissues. e ARMC1 was colocalizes with TIM23 in BRCA tissues. Scale bar: 12.5 $\mu \mathrm{m}$. ARMC1, Armadillo repeat-containing 1; BRCA, Invasive breast carcinoma; TOM20, the outer mitochondrial membrane 20; TIM23, the inner mitochondrial membrane 23; MCF-7, Human breast carcinoma cell lines; MCF-10A, Human normal breast cells.

\section{Supplementary Files}

This is a list of supplementary files associated with this preprint. Click to download.

- Supplement.docx 